\title{
The start of a new review series: recent advances in the management of secondary hypertension
}

\author{
Toshihiko Ishimitsu Editor-in-Chief ${ }^{1}$
}

Published online: 18 May 2020

(c) The Japanese Society of Hypertension 2020

The aging of society is an ongoing global phenomenon, occurring not only in the developed world but also in developing countries. In aging populations, the proportions of disease are naturally changing, and the numbers of patients suffering cardiovascular diseases including stroke and renal failure are increasing. Such cardiovascular diseases are based principally on the development of arteriosclerosis and atherosclerosis. Among the factors promoting arteriosclerosis, hypertension occupies a pivotal position as a modifiable risk. Therefore, the management of hypertension-namely, its prevention, detection, and treatment-is of primary importance to prevent the occurrence of cardiovascular diseases in modern societies.

In the etiology of hypertension, it is well known that essential hypertension is diagnosed in more than $90 \%$ of hypertensive patients, in which a specific cause is not identified other than the influences of aging, genetic predisposition, and unhealthy lifestyle. Table 1 lists the secondary causes of hypertension indicated in The Japanese Society of Hypertension Guidelines for the Management of Hypertension (JSH2019) [1]. Although the incidence of secondary hypertension is low compared with that of essential hypertension, its clinical importance should never be disregarded because of the following reasons. First, secondary hypertension is often curable by adequate surgical, interventional, and instrumental treatment of the causes which obviate the time and cost of long-term antihypertensive drug therapy. Second, secondary hypertension often presents resistance to the usual antihypertensive therapy, resulting in poor blood pressure control, which raises the risk of cardiovascular diseases and the development of cardiovascular organ failure. Therefore, the

Toshihiko Ishimitsu

isimitu@dokkyomed.ac.jp

1 Department of Nephrology and Hypertension, Dokkyo Medical University, Mibu, Japan
JSH2019 Guideline recommends investigating the possibility of secondary hypertension in cases such as juvenile hypertension, middle- to advanced-age-onset hypertension, severe hypertension, resistant hypertension, cases in which blood pressure control becomes difficult despite previous good control, rapid-onset hypertension, cases in which severe organ damage relative to the blood pressure level is present, and cases in which there are marked blood pressure fluctuations [1].

Among the causes of secondary hypertension shown in Table 1, it is a matter of debate as to the choice of interventional or drug therapy for renovascular hypertension considering the outcomes of recent clinical studies. As the results of advances in imaging technology and aggressive examination, it has become evident that the frequency of primary aldosteronism is higher than thought previously, and its appropriate management is being established. In addition, sleep apnea has been newly added to the list of

Table 1 Secondary causes of hypertension

Renovascular hypertension

Renal parenchymal hypertension

Primary aldosteronism

Sleep apnea

Pheochromocytoma

Cushing's syndrome

Subclinical Cushing's syndrome

Drug-induced hypertension

Aortic coarctation

Acromegaly

Hypothyroidism

Hyperthyroidism

Hyperparathyroidism

Brainstem vascular compression

Others

Adapted from [1] 
causes of secondary hypertension, as it causes sympathetic overactivity, and the application of continuous positive airway pressure therapy is effective in improving blood pressure control as well.

The series of review articles starting from this issue of Hypertension Research focuses on the new aspects of secondary hypertension such as newly recognized concepts, pathophysiology, epidemiology and the advancement of diagnostic procedures. Also addressed are surgical, interventional, instrumental, and medical treatment of renovascular hypertension, primary aldosteronism, pheochromocytoma, chronic kidney disease, and obstructive sleep apnea.

\section{Compliance with ethical standards}

Conflict of interest Honoraria: TI received honoraria from Takeda Pharmaceutical and a donation from Daiichi-Sankyo.

Publisher's note Springer Nature remains neutral with regard to jurisdictional claims in published maps and institutional affiliations.

\section{References}

1. Umemura S, Arima H, Arima S, Asayama K, Dohi Y, Hirooka Y, et al. The Japanese Society of hypertension guidelines for the management of hypertension (JSH 2019). Hypertens Res. 2019;42: $1235-481$. 